\title{
Atomic Resolution Imaging and Spectroscopy of Graphene Using the TEAM 0.5
}

Zonghoon Lee, ${ }^{*}$ Albert Dato,** Ki-Joon Jeon, ${ }^{* * *}$ Rolf Erni, ${ }^{*}$ Thomas J. Richardson, ${ }^{* * *}$ Michael Frenklach, ${ }^{* * * *}$ and Velimir Radmilovic*

* National Center for Electron Microscopy, Lawrence Berkeley National Laboratory, Berkeley, CA 94720

** Applied Science \& Technology Graduate Group, University of California, Berkeley, CA 94720

*** EETD, Lawrence Berkeley National Laboratory, Berkeley, CA 94720

**** Department of Mechanical Engineering, University of California, Berkeley, CA 94720

The free-standing graphene sheets, i.e. a two-dimensional (2D) single atomic sheet of carbon [1], were synthesized successfully from ethanol in a microwave plasma reactor [2]. The synthesized graphene layer, which represent highly ordered graphene sheets, is imaged successfully on the lately developed TEAM 0.5 instrument under the TEAM (Transmission Electron Aberration-Corrected Microscope) project [3]. The TEAM 0.5 is equipped with two aberration correctors for illumination and projection systems and with an electron monochromator, which have been optimized in 80-300 $\mathrm{kV}$. High resolution imaging of the graphene layers are very hard in any conventional microscopes because a higher $\mathrm{kV}$ beam damages the thinnest carbon layers and a lower $\mathrm{kV}$ operation deteriorates resolution for the light atoms. The TEAM instrument, however, brings us a high resolving power and sensitivity capability even at $80 \mathrm{kV}$ for imaging and spectroscopy of the preserved graphene layers. A TEM image from a typical region of the synthesized free-standing graphene layer is shown in Fig. 1 (A). Fig. 1 (B) displays densely packed hexagonal single-layer of carbon and unambiguous Moiré patterns formed from superimposed two individual graphene layers, which represent a coincidence site. Exit wave reconstructions were performed from a through-focus image series and the corresponding high resolution images were simulated using MacTempasX software for comparison. Monochromated electron energy loss spectra (EELS) of free-standing graphene sheets were also obtained. The carbon K-edge in EELS can be used to identify the structure of graphene sheets as shown in Fig.2. The EELS of single layer sheet exhibits unique graphitic characteristics, which has the $1 \mathrm{~s}-\pi^{*}$ and $1 \mathrm{~s}-\sigma^{*}$ transition at $285 \mathrm{eV}$ and $291 \mathrm{eV}$.

It is also challenging to distinguish fine-scale single-layer graphene from other carbon nanostructures. We are able to perform STEM parallel beam diffraction on nano region of graphene single-layer and bilayer using a Zeiss Libra 200 FE-TEM operated at 200kV with Koehler illumination. The several sets of diffraction patterns from single graphene layer and two layers of graphite appear differently because the synthesized graphene sheets exist typically in overlapped and folded sheets on TEM sample grids. A set of hexagonal diffraction spots from a single graphene layer can be identified by simulated diffraction patterns as shown in Fig. 3 [4].

References

[1] K. S. Novoselov et al., Science 306 (2004) 666.

[2] A. Dato et al., Nano Letters 8 (2008) 2012.

[3] The TEAM project (http://ncem.lbl.gov/TEAM-project/index.html).

[4] J. C. Meyer et al., Nature 446 (2007) 60.

[5] The work was supported by the National Aeronautics and Space Administration, grant \#NCC3-

833 and the U.S. Department of Energy under Contract \# DE-AC02-05CH11231. 

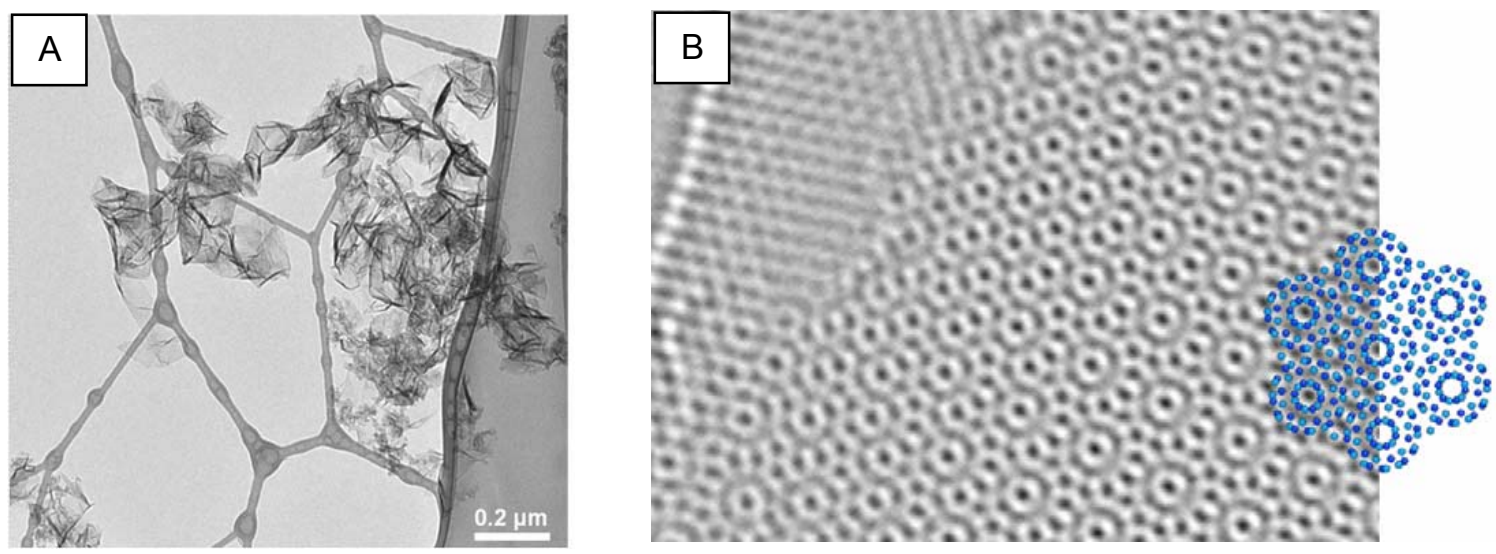

Fig. 1. (A) A typical TEM image of synthesized graphene sheets. (B) An exit wave reconstructed image of single-layer and bilayer graphene along with superimposed carbon atom positions.
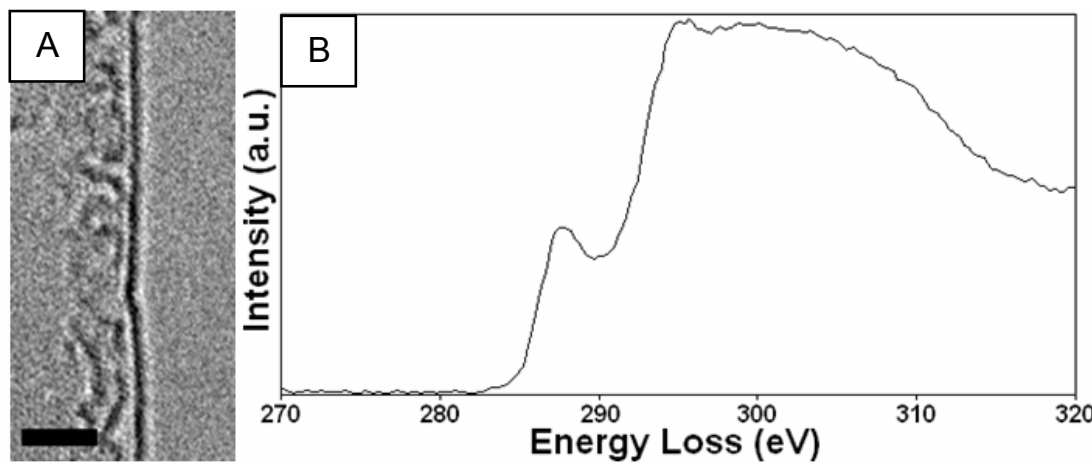

Fig. 2. (A) TEM image of single layer graphene and (B) corresponding EELS spectra taken from the single layer sheet. Scale bars, $2 \mathrm{~nm}[2]$.
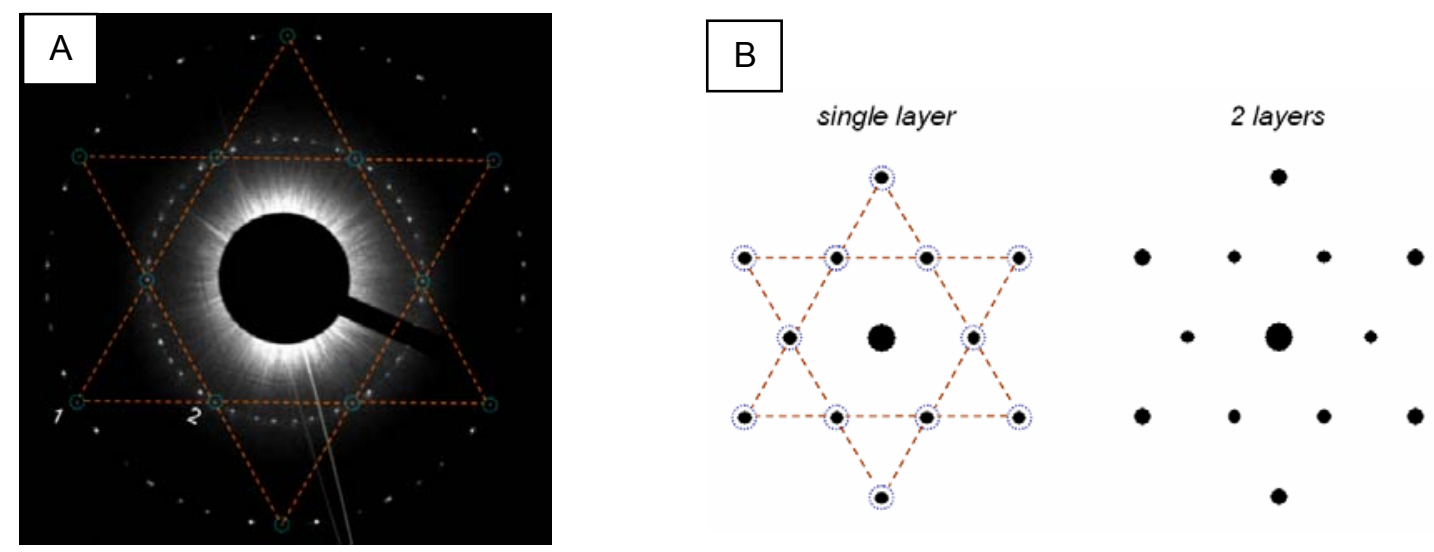

Fig. 3. (A) An electron diffraction pattern from a region containing several overlapped single layer and bilayer graphene sheets. The diffraction spots from single layer graphene are indicated with circles. (B) Simulated diffraction patterns of a single layer and bilayer graphene. Careful intensity measurements of diffraction spots along a line connecting points 1 and 2 reveals single-layer graphene. 